\title{
Components of Young Children's Trait Understanding: Behavior-to-Trait Inferences and Trait-to-Behavior Predictions
}

\author{
David Liu, Susan A. Gelman, and Henry M. Wellman \\ University of Michigan
}

\begin{abstract}
Trait attribution is central to people's naïve theories of people and their actions. Previous developmental research indicates that young children are poor at predicting behaviors from past trait-relevant behaviors. We propose that the cognitive process of behavior-to-behavior predictions consists of two component processes: (1) behavior-totrait inferences and (2) trait-to-behavior predictions. Experiment 1 demonstrates that 4-, 5-, 7-, and 9-year-olds can infer trait labels from behaviors. Experiment 2 demonstrates that 4-, 5-, and 7-year-olds can predict behaviors from trait labels but not from past behaviors. Experiment 3 demonstrates that 4- and 5-year-olds understand traits as predictive and stable over time. Taken together, these three studies show that young children, in possessing component trait-reasoning processes, have a nascent understanding of traits.
\end{abstract}

Developing a commonsense understanding of people and their actions is necessary for success in complex social interactions, meaningful relationships, cultural learning, and self-perception. As such, critical to our successful navigation of the social world is the possession of elegant social cognitive abilities (Adolphs, 1999; Tomasello, 1999; Whiten \& Byrne, 1997). Of late, research in children's developing social cognition has focused on how they acquire a theory of mindunderstanding that people's behaviors are causally related to internal mental states and attributing such mental states (Wellman, 1990). However, beyond theory of mind, human social cognition consists of other important understandings and abilities. One significant aspect of adults' everyday folk psychology is reasoning about dispositional traits (Heider, 1958; Kelley, 1973; Weiner, 1974). Trait attribution is central to much of our naïve theories of the social world, in our everyday explanations and predictions of people's behaviors and thoughts (Jones, 1979; Nisbett \& Ross, 1980; Trope \& Higgins, 1993). Researchers also consider children's trait conceptions important for issues of achievement motivation (Cain \& Dweck, 1995; Dweck, Hong, \& Chiu, 1993; Heyman \& Dweck, 1998) and social interactions (Camhy \& Ruble, 1994; Erdley \& Dweck, 1993). Nevertheless, much is still

Funding for this research was provided by a NSF Graduate Fellowship to Liu, a NICHD grant HD-36043 to Gelman, and a NICHD grant HD-22149 to Wellman. We thank the children, parents, and the staff of the University of Michigan Children's Centers, Ann Arbor YMCA Preschool Program, St. Thomas School, and St. Francis School for their participation.

Correspondence concerning this article should be sent to David Liu, now at the Institute for Learning and Brain Sciences, University of Washington, Box 357988, Seattle, WA 98195-7988. Electronic mail may be sent to davidliu@u.washington.edu. unknown about how children come to a conception of traits similar to that of adult folk psychology.

Dispositional trait understanding involves the attribution to the self or others of stable psychological characteristics (Rotenberg, 1980). To distinguish traits from other folk psychological concepts, it is important to clarify that trait attribution characterizes a personal, psychological quality as being both internal and stable across time and situations. One can attribute internal, mental characteristics, such as someone's desire, without it being a trait, because desire is not necessarily a stable disposition (Wellman, 1990). Similarly, one can attribute stable characteristics, such as someone's height, without it being a trait, because height is not an internal psychological disposition. Full dispositional conceptualization entails inferring an internal psychological characteristic and understanding its stability across time and situations in causing behaviors.

The general claim from developmental research is that not until around 7 to 8 years of age (i.e., middle childhood) do children begin to understand the stable, internal, and causal nature of traits (for reviews, see Flavell \& Miller, 1998; Miller \& Aloise, 1989; Ruble \& Dweck, 1995; Yuill, 1992). This is evident especially in a dramatic developmental shift in children's appreciation and use of traits to predict and explain people's behaviors (Rholes \& Ruble, 1984; Rholes, Newman, \& Ruble, 1990). Early research observed that children under the age of 8 years rarely used trait terms to describe themselves or others, whereas older children frequently used trait terms in these situations (Barenboim, 1981; Livesley \& Bromley, 1973; Peevers \& Secord, 1973). Instead,

(C) 2007 by the Society for Research in Child Development, Inc. All rights reserved. 0009-3920/2007/7805-0009 
young children tended to describe themselves and others in more superficial terms, such as actions, appearances, possessions, and places of residence. More controlled experimental paradigms that examined children's trait-consistent predictions of behavior also found a developmental shift around 7 to 8 years of age in trait reasoning (Berndt \& Heller, 1986; Ferguson, Van Roozendaal, \& Rule, 1986; Kalish, 2002; Rholes \& Ruble, 1984; Rotenberg, 1980, 1982). These experiments addressed the extent to which young children comprehend the stable, causal nature of traits, by asking them to predict behaviors given trait information. Consider the study by Rholes and Ruble (1984), where kindergartners (5- and 6-year-olds) and fourth graders (9- and 10-year-olds) were presented with videos of individual protagonists engaging in trait-relevant behaviors (e.g., a girl who is sharing her lunch). The experimenters then asked these children to predict which of two behaviors the protagonists would perform in the future in a new situation (e.g., helping or not helping her friend rake leaves). Only the older children correctly made trait-consistent predictions of behavior. Together, these findings have led to the conclusion that preschool-age children do not understand traits as stable, mediating dispositions.

Children's seemingly late development in trait conceptualization is in line with the Piagetian claim of younger children reasoning in superficial and situational terms and older children reasoning in causal, internal, and stable terms (Flavell, 1977; Livesley \& Bromley, 1973; Rholes et al., 1990; Shantz, 1983). However, this characterization of cognitive development is considered outdated in light of recent research in children's theory of mind and essentialism (see Flavell \& Miller, 1998; Gelman, Coley, \& Gottfried, 1994; Taylor, 1996; Wellman \& Gelman, 1998) that depicts young children's robust understanding of internal psychological states. Thus, by 3 and 4 years of age children predict and explain behaviors in terms of internal mental entities such as desires and beliefs (Wellman, 1990; Wellman, Cross, \& Watson, 2001). In some studies, infants and toddlers have demonstrated an appreciation of the subjective nature of desires (Repacholi \& Gopnik, 1997; Wellman \& Liu, 2004; Wellman \& Woolley, 1990) and a conception of the intentions behind goal-directed actions (Carpenter, Akhtar, \& Tomasello, 1998; Gergely, Nadasdy, Csibra, \& Biro, 1995; Meltzoff, 1995). In contrast to the Piagetian claim, this more recent research has shown that young children do not necessarily focus on superficial or situational characteristics of actions, penetrating instead to deeper, psychological states and features.
Previous experimental studies on children's trait understanding have overwhelmingly asked young children to predict trait-consistent behaviors from trait-relevant behaviors, just as in the Rholes and Ruble (1984) study outlined earlier. As Heyman and Gelman (1999) pointed out, children in these studies were asked to make property-to-property inferences, instead of property-to-category inferences (i.e., infer traits from behaviors) or category-to-property inferences (i.e., predicting behaviors from traits). Presumably when older children succeed in these studies they are making a property-to-category inference (infer a trait from behavior) and a category-to-property prediction (predict behavior from that trait). When younger children fail, perhaps they know nothing about traits or, alternatively, perhaps they know something about traits but have difficulty with one or both parts of this two-part inference process. In the current studies, we examined whether preschool-age children are capable of the component processes of behavior-to-trait inferences and trait-to-behavior predictions, despite having difficulty with behavior-tobehavior predictions. We hypothesized that when the tasks are decomposed in this fashion young children would reveal important initial trait understandings. In Experiment 1 we examined behavior-to-trait inferences; in Experiments 2 and 3 we examined trait-tobehavior predictions.

\section{Experiment 1}

Following our analysis of behavior-to-behavior predictions as consisting of two component processes of behavior-to-trait inferences and trait-to-behavior predictions, Experiment 1 investigated the first component process-namely, behavior-to-trait inferences. Past studies have shown that young children are able to appropriately assign trait labels to provided examples of behavior (Rholes \& Ruble, 1984; Stipek \& Daniels, 1990). However, researchers have suggested that what preschoolers are doing, rather than understanding traits, is simply valence-based evaluations of people's behaviors (Alvarez, Ruble, \& Bolger, 2001; Rholes et al., 1990; Ruble \& Dweck, 1995). That is, young children make egocentric and global evaluations about whether they like or dislike a person and then make judgments and predictions from their evaluations. In a study by Alvarez et al. (2001), children were provided with vignettes of protagonists engaging in trait-relevant behaviors and were then asked to evaluate the goodness of the characters, to rate the characters on relevant traits, and to predict future behaviors. They found, with path analysis, that 
although older children's trait ratings mediated their predictions, younger children's evaluative ratings mediated their predictions. This suggests that young children use global evaluations to make what can appear to be trait inferences. Similarly, Ruble and Dweck (1995) suggest that young children could have an appreciation of people as either good or bad. This hypothesis posits a superficial understanding that merely divides and categorizes people globally without alluding to internal, causal characteristics. Thus, superficial evaluations could lead young children to appropriately assign trait labels to examples of behavior (Rholes \& Ruble, 1984; Stipek \& Daniels, 1990) without an ability to infer traits from behaviors (i.e., property-to-category inferences). To test this, in Experiment 1 , we presented children with story protagonists' trait-relevant behaviors and then asked them to make trait-label judgments and ratings. We also asked children to rate how much they liked the protagonists, in order to determine whether simple evaluative ratings mediated their trait labeling. It is possible that even if young children do use global evaluations to make trait inferences, this does not preclude them from understanding traits. Young children, and adults, may have a conception of traits but sometimes rely on evaluative heuristics to make inferences, especially when the traits involved are highly valenced.

Furthermore, the majority of previous research testing behavior-to-behavior predictions has presented children with only one example of a story protagonist's trait-relevant behavior. For instance, children were shown a story character sharing her lunch and from that single example of trait-relevant behavior, children were expected to make predictions of future behavior. It is well documented that adults, at least in Western cultures, have a strong tendency to attribute another person's behavior to internal traits and ignore situational factors even in the absence of any evidence for the stability of that behavior (for reviews, see Gilbert, 1998; Gilbert \& Malone, 1995). This is argued by social psychologists to be the "fundamental attribution error" (Jones, 1979; Nisbett \& Ross, 1980; Ross, 1977). It is exactly this type of "error," of making trait attributions from a single sample of behavior, that the standard study design demands of children. From these sorts of data, it may be the case that young children understand traits, but (before 8 years of age) they do not make one-example behavior trait attributions (see, e.g., Miller, 1984). Instead, they might require multiple examples of behavior rather than just one in order to make a trait attribution (Boseovski \& Lee, 2006). In their everyday life, children often observe people behave consistently and repeatedly over time; repeated examples may be more naturalistic and effective in engaging young children's inferences of traits from behavior. Thus, we assessed whether young children would be better at behavior-to-trait inferences with three examples than with one example of trait-relevant behavior.

Each child made inferences to four possible traits (selfish, nice, mean, and shy) by making judgments about a story character behaving in trait-consistent or trait-inconsistent ways. For example, for judgments of selfishness, a trait-consistent story described a character's behavior of not sharing his lunch, and a traitinconsistent story described a character's behavior of sharing his lunch. In each case, we then asked children to make a trait-label judgment (e.g., "Is Bobby selfish?"). If children understand the link between behaviors and traits, they should differentially judge the selfishness of a story character not sharing his lunch versus a story character sharing his lunch.

We also asked children to rate how much they liked the protagonist, to be used as a covariate in the analysis in order to determine whether children's behavior-totrait judgments went beyond global evaluations and reflected deeper inferences. We further addressed the global evaluation hypothesis by including the "shy" trait, because it is not as valenced as selfishness, niceness, or meanness. It is unclear whether children evaluate shyness positively, negatively, or neither. However, if they judge it more neutrally, then by studying children's understanding of such a "neutral" trait, we can examine whether young children have conceptions of traits that do not simply reduce to global good/bad or like/dislike evaluations.

\section{Method}

\section{Participants}

Sixty-four children, 4-, 5-, 7-, and 9-year-olds, from a small Midwestern American city participated in Experiment 1. The sample consisted of 16 children (8 boys and 8 girls) from each age group. The mean age (and range) in years-months for each age group was 46 (4-0 to 4-11), 5-8 (5-0 to 6-3), 7-9 (7-2 to 8-6), and 9-9 (9-2 to 10-4). The children came from preschools and elementary schools serving a middle-class population that was largely European-American with approximately 25\% Asian-, African-, and Latino-American representation.

\section{Design}

Each child heard four stories, one for each of four traits: selfish, nice, mean, and shy. The main 
manipulation was whether the protagonist engaged in trait-consistent behaviors or trait-inconsistent behaviors. This manipulation was within subjects. Comparisons of children's trait-label judgments and ratings between the trait-consistent condition (two stories) and the trait-inconsistent condition (two stories) revealed whether children made judgments and ratings differentially between the two conditions and, thus, indicated whether children correctly inferred trait labels from behaviors. For each protagonist, children also made an evaluative judgment and rating. Furthermore, we presented two of the stories with one example of trait-relevant behavior in each story, and we presented the other two stories with three examples of trait-relevant behavior in each story. The order of presentation of the four traits, the order of the traitconsistent and trait-inconsistent conditions, and the order of the one-example and three-examples conditions were counterbalanced.

\section{Materials}

Each of the four stories presented to the children contained a different protagonist (see stories in Appendix A). To rule out gender bias in children's trait attributions, the story protagonists were sex matched with each child participant, and all the other characters in the stories were of the other sex.

For ease of comprehension, we structured all stories in a consistent manner with the same template. It is important to note that the stories were purposely and strictly designed to avoid the use of mental state terms (e.g., want, like, or help). The stories only described settings and behaviors. We did this so that any trait inferences the children made from the stories were from the protagonists' behaviors and not simply from the valance of the terms used to describe the behaviors. Pictures to help with comprehension accompanied the presentation of the stories. In all of the pictures, story protagonists' faces were turned away to control against children using facial expressions, and not trait understanding, to form their trait inferences. Therefore, any trait inferences the children made from the stories and pictures were from the protagonists' behaviors and not from facial expressions.

\section{Procedure}

Children were tested individually. After presentation of each story, children were asked to judge whether the story protagonist was of a certain trait label (e.g., "Is Bobby selfish?"). If the judgment was in the affirmative (i.e., responding "yes"), in any condi- tion (trait-consistent and trait-inconsistent), we then asked the children to rate that trait on a 3-point scale (e.g., "Is Bobby a little selfish, medium selfish, or very selfish?"); this was accompanied by a visual scale (three progressively larger blue squares on a piece of paper) to help comprehension. This resulted in a 4point rating scale of that trait label: a score of 0 for judging no, a score of 1 for judging yes and rating a little, a score of 2 for judging yes and rating medium, and a score of 3 for judging yes and rating very. We first considered children's yes/no judgment, which is a common methodology in cognitive development research with young children, but we also obtained a more detailed measurement with the 4-point rating scale. As will be presented below, both measures converged in the results.

In addition, we asked children to judge whether he or she liked the story protagonist (e.g., "Do you like Bobby?"). If the judgment was in the affirmative, we then asked the children to rate how much they liked the story protagonist on a 3-point scale (e.g., "Do you like Bobby a little, medium, or a lot?"), accompanied by another visual scale (three progressively larger yellow circles on a piece of paper) to help comprehension. The evaluative rating scale was scored in the same manner as the trait-label rating scale, by combining the evaluative judgment and the 3-point scale to create a 4-point evaluative rating scale of that story protagonist.

\section{Results}

For all measures-trait-label judgments, trait-label ratings, and evaluative ratings - an initial 2 (examples: one-example vs. three-examples) $\times 4$ (age group: 4-, 5-, 7-, and 9-year-olds) repeated measures analysis of variance (ANOVA) was conducted. The results showed that each of these measures did not differ between presentation of one example of trait-relevant behavior and presentation of three examples of traitrelevant behavior, all $p \mathrm{~s}>.70$. In addition, there were no interaction effects between examples and age, all $p s>.40$. Therefore, we collapsed our measures across one example and three examples of trait-relevant behavior for all further analyses.

For trait-label judgments and trait-label ratings, a 2 (trait presentation: trait-consistent vs. trait-inconsistent) $\times 4$ (age group: 4-, 5-, 7-, and 9-year-olds) repeated measures ANOVA was conducted. As shown in Figures 1 and 2, trait-label judgments (which range from a score of 0 to 2 ) and trait-label ratings (which range from a score of 0 to 6) differed significantly depending on trait presentation condition (traitconsistent vs. trait-inconsistent), $F(1,60)=515.00$, 


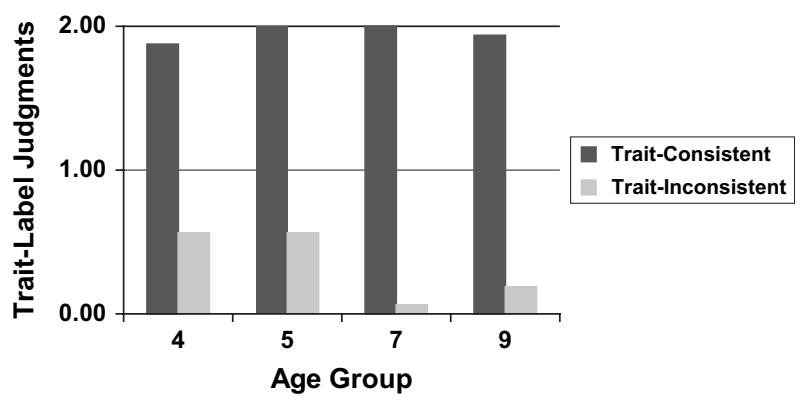

Figure 1. Trait-label judgments by age and trait presentation conditions (trait-consistent vs. trait-inconsistent) in Experiment 1.

$p<.001$, and $F(1,60)=590.40, p<.001$, respectively. This difference was observed for all four traits; the mean trait-label ratings for trait-consistent and traitinconsistent conditions were, respectively, 2.72 and 0.25 for selfish, 2.81 and 0.19 for nice, 2.63 and 0.88 for mean, and 2.69 and 0.31 for shy. In addition, there were interaction effects between trait presentation and age for trait-label judgments and for trait-label ratings, $F(3,60)=4.06, p<.05$, and $F(3,60)=5.94, p<$ .01 , respectively. It appears that the ability to correctly judge and rate traits differentially for different behaviors improves with age (see Figures 1 and 2). Nevertheless, examined separately, all age groups showed significant differentiation in their trait-label judgments and trait-label ratings depending on trait presentation condition, including the 4-year-olds' judgments and ratings, $F(1,15)=55.59, p<.001$, and $F(1,15)=47.97, p<.001$, respectively. These results with both measures demonstrated that even the 4-year-olds were able to infer traits from behaviors (e.g., when 4-year-olds were presented with the story of Bobby not sharing his cookies, they judged that Bobby is selfish, but when 4-year-olds were presented with the story of Bobby sharing his cookies, they judged that Bobby is not selfish). Therefore, although there was a developmental trend in children's judgments, even children from the youngest age tested were quite able to make behavior-to-trait inferences.

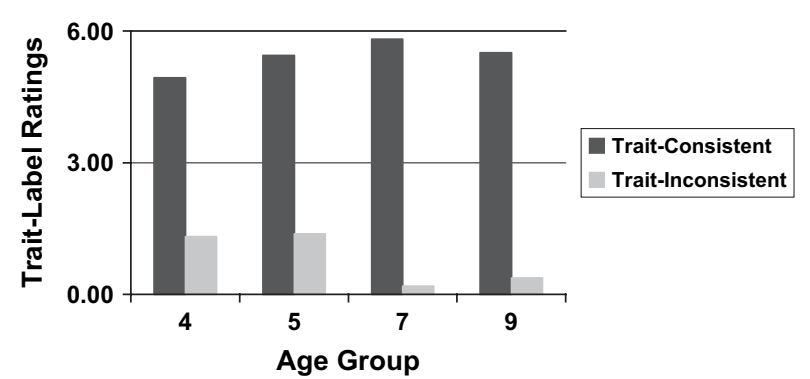

Figure 2. Trait-label ratings by age and trait presentation conditions (trait-consistent vs. trait-inconsistent) in Experiment 1.
Next, we examined whether young children's behavior-to-trait inferences were solely mediated by evaluative ratings. Not surprisingly, evaluative ratings were significantly more positive for the notselfish story than for the selfish story, $F(1,56)=$ $100.69, p<.001$, for the nice story than for the notnice story, $F(1,56)=54.51, p<.001$, and for the notmean story than for the mean story, $F(1,56)=13.22$, $p<.01$. There was an interaction effect between trait presentation and age for selfish evaluative rating, $F(3$, $56)=3.46, p<.05$. There were no interaction effects with age group for nice and mean evaluative ratings, all $p s>.10$. However, of central importance, as shown in Figure 3, evaluative ratings for the shy story and for the not-shy story did not differ significantly, $F(1,56)=$ $3.81, n s$, and there was no interaction effect with age group, $F(3,56)=1.17$, ns. These results demonstrated that children made consistent global evaluations for selfishness, niceness, and meanness, but did not do so for shyness. Children did not evaluate shy behavior more or less positively than not-shy behavior. Shyness appears to be a more "neutral" trait than selfishness, niceness, and meanness for children. Therefore, our demonstration of early understanding of shyness argues against a strictly global, evaluative interpretation of young children's trait understanding.

Trait-label ratings and evaluative ratings were significantly correlated for three of the traits: selfishness, $r(64)=-.72, p<.001$, niceness, $r(64)=.77$, $p<.001$, and meanness, $r(64)=-.60, p<.001$. However, trait-label rating and evaluative rating for shyness were not correlated, $r(64)=-.10, n s$. It seems that the children did not use their evaluative rating to infer shyness from behavior. Furthermore, even when evaluative ratings for each of the four traits were included as a covariate in an analysis of covariance (ANCOVA) for each individual trait's trait-label ratings, all four trait-label ratings still differed significantly depending on trait presentation condition: selfishness, $F(1,55)=66.55, p<.001$, niceness, $F(1,55)=114.44, p<.001$, meanness, $F(1,55)=27.51$,

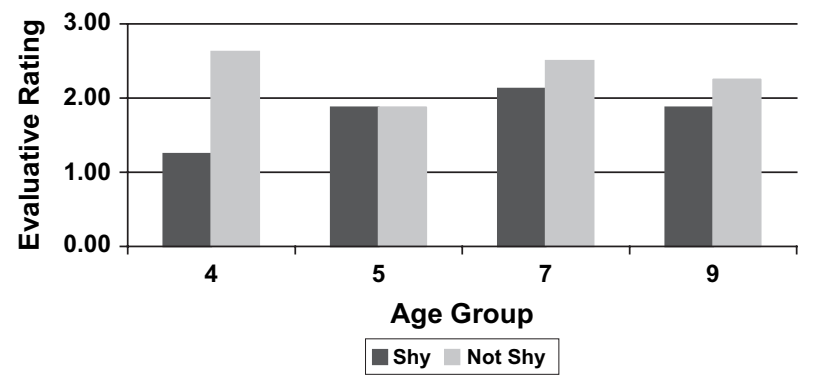

Figure 3. Evaluative ratings for shyness by age and trait presentation conditions in Experiment 1. 
$p<.001$, and especially shyness, $F(1,55)=165.68, p<$ .001 . Across the different traits, evaluative ratings did not account for all of the variance in children's traitlabel ratings. Therefore, these results strongly suggest that evaluative ratings do not completely mediate children's trait-label inferences.

\section{Discussion}

Young children were able to infer trait labels from behaviors. In addition, the number of trait-relevant example behaviors did not have a significant effect on trait-label judgments or trait-label ratings. This, however, could be because children's performance was already at high levels when given one example of behavior. For the four traits we presented, we took pains to focus on traits that might be among those that children first understand. If so, it is possible that with other, harder traits (e.g., pessimism, extraversion, etc.) the more naturalistic condition of providing multiple examples of a character's trait-relevant behavior would make it easier for young children to make trait-consistent inferences (see Boseovski \& Lee, 2006).

Even children from the youngest age tested, 4-yearolds, showed an ability to make behavior-to-trait inferences that were not solely mediated by global evaluations, as evident in the covariance analyses. More importantly, although there were consistent global evaluations associated with selfishness, niceness, and meanness, shyness appears to be a more "neutral" trait. Children's shyness ratings did not correlate with their evaluative ratings. Therefore, the demonstration in Experiment 1 of young children's trait understanding for shyness serves as a case against a purely global, evaluative interpretation of young children's trait understanding.

Experiment 1 thus provides support for young children being capable of the first component process of behavior-to-trait inferences, at least when asked to make such inferences. Experiment 2 investigated young children's capacity for our proposed second component process of trait-to-behavior predictions.

\section{Experiment 2}

Previous research using behavior-to-behavior prediction tasks suggests that preschool-age children have clear difficulty using traits to predict behaviors (e.g., Rholes \& Ruble, 1984). Yet there is some evidence that preschool-age children are able to use traitrelevant information to make other sorts of predictions and inferences (Cain, Heyman, \& Walker, 1997; Eder,
1989, 1990; Gnepp \& Chilamkurti, 1988; Heller \& Berndt, 1981; Heyman \& Gelman, 1999, 2000; Stipek \& Daniels, 1990; Yuill \& Pearson, 1998). For instance, Heyman and Gelman (1999) found that when preschool-age children were told that a trait label of "mean" applied to a story protagonist who sprayed another character with water, they correctly predicted that the protagonist would be upset when it turned out that the other character was actually happy to get sprayed. For the most part, these studies show that what younger children can infer from trait information are mental states, such as motivation and emotion. That preschool-age children, to the extent they make use of trait information, are able to infer traitrelated mental states is in keeping with their already formed theory of mind (see also Heyman \& Gelman, 2000). What about young children's ability or inability to use traits to predict behavior?

Given the results from Experiment 1 (and previous research, e.g., Rholes \& Ruble, 1984) showing that young children have some comprehension of trait labels, as well as the results from a few studies showing children's ability to use trait labels to make psychological inferences (Heyman \& Gelman, 1999, 2000), young children may also be able to use trait labels to predict behavior. Specifically, we hypothesized that young children would be able to predict trait-consistent behaviors when given trait labels (trait-to-behavior predictions). Moreover, they would be better at predicting behaviors when given trait labels than when given examples of trait-relevant behavior. Inferring future behaviors from past traitrelevant behaviors constitutes behavior-to-behavior predictions, and, as discussed above, past research indicates that young children would be poor at such predictions.

In Experiment 2, each child participated in four parallel trials, one for each of four traits (selfish, nice, mean, and shy). In a within-subjects design, we presented children with a trait label for two of the trials and an example of trait-relevant behavior for the other two trials. After the children were presented with the trait information in each trial, we asked children to predict whether in a new situation the protagonist would behave in a manner consistent with the trait or in a manner inconsistent with the trait.

\section{Method}

\section{Participants}

Forty-eight children, 4-, 5-, and 7-year-olds, from a small Midwestern American city participated in 
Experiment 2 (these children did not participate in Experiment 1). The sample consisted of 16 children (8 boys and 8 girls) in each age group. The mean age (and range) for each age group was 4-6 (4-0 to 4-11), 56 (5-0 to 6-0), and 7-5 (6-11 to 7-11). The children came from preschools and elementary schools serving a middle-class population that was largely EuropeanAmerican with approximately 25\% Asian-, African-, and Latino-American representation.

\section{Materials}

In the current experiment, we again tested four traits: selfish, nice, mean, and shy. The main manipulation involved presenting either trait labels or traitrelevant behaviors. Comparisons of children's predictions between these two trait-information conditions tested whether children were more likely to make traitconsistent behavioral predictions when given trait labels than when given examples of trait-relevant behavior.

For the trait-relevant behaviors condition, we used four of the trait-consistent stories from Experiment 1 , one for each trait (the first trait-consistent behavior of each trait in Appendix A for Experiment 1 was the exact behavior also used in Experiment 2). Each story involved a different protagonist. As in Experiment 1, the story protagonists were sex matched with each child participant and all the other characters in the stories were of the other sex. For the trait labels condition, child participants were told that protagonists possessed particular traits (e.g., "This is Bobby and he is selfish. Bobby is a very selfish boy."). Pictures to help with comprehension accompanied the presentation of the stories. In all of the pictures, story protagonists' faces were turned away to control against children using facial expressions instead of trait understanding to form their predictions.

\section{Procedure}

Children were tested individually. For each trait, after presenting either a trait label or a trait-relevant behavior, we assessed children's memory of the trait information to ensure comprehension. Next, we told children of a trait-relevant situation and asked them to predict the protagonist's behavior (e.g., "One day Bobby sees his younger sister playing with his toys in his room. What does Bobby do when he sees his sister playing with his toys?"). We provided children with a choice between a trait-consistent behavior and a trait-inconsistent behavior that were opposites of each other (e.g., "Does he stop her from playing with his toys? Or does he let her keep playing and give her more of his toys to play with?"). See Appendix B for the trait-relevant situation and prediction choices for each trait.

\section{Results}

A 2 (trait information: trait labels vs. trait-relevant behaviors) $\times 3$ (age group: 4-, 5-, 7-year-olds) repeated measures ANOVA was conducted. As shown in Figure 4, children were significantly better at traitconsistent predictions when given trait labels than when given trait-consistent behaviors, $F(1,45)=$ $10.40, p<.01$. There was neither a main effect nor an interaction effect with age group. The lack of an interaction with age confirms that children in all age groups were better given trait labels than trait behavior. Targeted analyses of 4- and 5-year-olds showed that 5-year-olds were significantly better at traitconsistent predictions from trait labels than from trait-relevant behaviors, $F(1,15)=5.45, p<.05$; 4-year-olds were also better at trait-consistent predictions from trait labels than from trait-relevant behaviors, but the effect was not significant.

Both 4- and 5-year-olds were better than chance at predictions from trait labels. Specifically, because we provided children two choices for their predictions, chance performance in each condition, which involved two trials, was 1.00 out of the maximum score of 2.00. Examining these age groups together with one-sample $t$-tests against the chance value of 1.00 , predictions from trait labels were significantly greater than chance, $t(31)=3.04, p<.01$, whereas predictions from trait-relevant behaviors were not significantly greater than chance. That predictions from trait-relevant behaviors were no better than chance is consistent with the literature on young

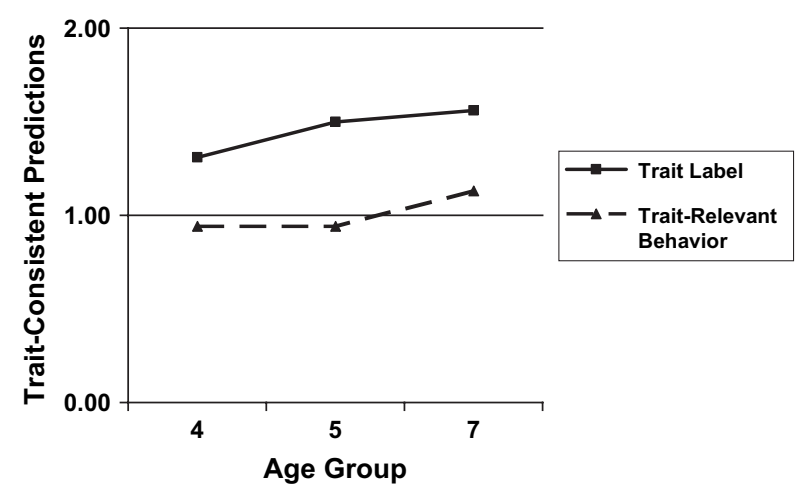

Figure 4. Trait-consistent predictions by age and trait presentation conditions (trait labels vs. trait-relevant behaviors) in Experiment 2. 
children's (including 7-year-olds') inconsistency in using trait information to predict behaviors (e.g., Rholes \& Ruble, 1984).

\section{Discussion}

In Experiment 2, preschool-age children were consistent in making trait-consistent behavioral predictions when presented with trait labels, whereas they were inconsistent in making trait-consistent behavioral predictions when presented with traitrelevant behaviors. This finding for behavior-tobehavior predictions is consistent with previous research, which suggests that children of this age have difficulty with predicting behaviors (Kalish, 2002; Rholes \& Ruble, 1984). However, our results showed that these same children were able to make the component process of trait-to-behavior predictions. Specifically, when the task entails traitto-behavior predictions, rather than behavior-tobehavior predictions, we demonstrate, for the first time, an aspect of predicting behaviors from trait information that they are capable of. Indeed, across Experiments 1 and 2 young children demonstrate an ability to perform both the component processes of behavior-to-trait inferences and trait-to-behavior predictions. Yet they were inconsistent for behaviorto-behavior predictions. Apparently although they are able to operate with both component processes separately (and thereby revealing a useful, appropriate understanding of traits), preschool-age children have yet to link these two component processes together for a full and sophisticated conception of traits that involves trait-consistent behavior-tobehavior predictions.

Even without being able to make behavior-tobehavior predictions, trait-to-behavior predictions of the sort we demonstrate in Experiment 2 could be important and useful to young children. In everyday life, not all of our knowledge about other people comes from direct observation of their behaviors. We also talk about each other all the time, as in gossip (see Dunbar, 1993, and associated commentary for a discussion on the central role of gossip-estimated as $60 \%$ of everyday human conversation-in the evolution of language). Much of what we know about people comes from what others tell us, and this is often done using trait labels. For instance, if people tell us that a particular person is "shy," we will have certain expectations of that person's behavior in social situations full of strangers. Experiment 2 suggests that young children can begin to participate in traitrelevant discourse and, based on such information, make trait-consistent behavioral predictions.

\section{Experiment 3}

We propose that in making trait-to-behavior predictions, young children operate with a notion of traits as predictive and stable. Experiment 3 more fully tests children's understanding of stability across time. Whereas children in Experiment 2 were only asked to provide behavioral predictions for one time point, children in Experiment 3 were asked to provide predictions for two distinct time points. As in Experiment 2, each child participated in four parallel trials, one for each of four traits (selfish, nice, mean, and shy). In a within-subjects design, we presented children with a trait label for two of the trials and an example of trait-relevant behavior for the other two trials. After the children were presented with the trait information in each trial, we asked children to predict whether the protagonist would, at two future time points, behave in a manner consistent with the trait or in a manner inconsistent with the trait. No previous study on young children's trait understanding has actually directly assessed predictions across multiple time points. However, if children conceive of traits as stable and predictive, then they should make traitconsistent predictions for multiple time points. We hypothesized that, for Time 1 and for Time 2, children would make trait-consistent predictions and would be better at predicting behaviors when given trait labels than when given examples of trait-relevant behavior.

\section{Method}

\section{Participants}

Thirty-two children, 4- and 5-year-olds, from a small Midwestern American city participated in Experiment 3. None of these children participated in Experiment 1 or Experiment 2. The sample consisted of 16 children ( 8 boys and 8 girls) in each age group. The mean age (and range) for each age group was 4-8 (4-2 to 4-11) and 5-4 (5-0 to 5-11). The children came from preschools serving a middle-class population that was largely European-American with approximately 25\% Asian-, African-, and Latino-American representation.

\section{Materials and Procedure}

The materials and procedure used in Experiment 3 were the same as those used in Experiment 2. However, in Experiment 3, for each of the four traits, there was an added question asking children to predict the protagonist's behavior at Time 2, in addition to Time 1. For Time 1, we asked children to predict the 
protagonist's behavior "one day" using the same wording as in Experiment 2 (e.g., "One day, Bobby sees his younger sister playing with his toys in his room. What does Bobby do when he sees his sister playing with his toys?"). For Time 2, we asked children to predict the protagonist's behavior a second time "a month later" in a new setting when faced with the same situation (e.g., "One day a month later, Bobby again sees his younger sister playing with his toys in the basement. What does Bobby do when he sees his sister playing with his toys?").

\section{Results}

\section{Time 1 Predictions}

A 2 (trait information: trait labels vs. trait-relevant behaviors) $\times 3$ (age group: 4-, 5-year-olds) repeated measures ANOVA was conducted for children's response for Time 1; a parallel repeated measures ANOVA was conducted for children's response for Time 2. Replicating the results of Experiment 2, children in Experiment 3, as shown in Figure 5a, were significantly better at Time 1 predictions when given trait labels than when given trait-consistent behaviors, $F(1,30)=10.68, p<.01$. In addition, there was neither a main effect nor an interaction effect with age group. Targeted analyses of 4- and 5-year-olds separately showed that 4-year-olds were significantly better at trait-consistent Time 1 predictions from trait labels than from trait-relevant behaviors, $F(1,15)=$ $5.95, p<.05$. Similarly, 5-year-olds were significantly better at trait-consistent Time 1 predictions from trait labels than from trait-relevant behaviors, $F(1,15)=$ $4.77, p<.05$. As in Experiment 2, because we provided children two choices for their predictions, chance performance in each condition, which involved two trials, was 1.00 out of the maximum score of 2.00. The results showed that 4-year-olds' Time 1 predictions from trait labels were significantly greater than chance, $t(15)=2.74, p<.05$, whereas their predictions from trait-relevant behaviors were not significantly different from chance; 5 -year-olds' Time 1 predictions from trait labels were also significantly greater than chance, $t(15)=3.09, p<.01$, whereas their predictions from trait-relevant behaviors were not significantly different from chance.

Furthermore, children's individual patterns of response support these results. It was the case that out of two trials most 4- and 5-year-olds were consistent for predictions from trait labels and were inconsistent for predictions from trait-relevant behaviors. Over two trials, by chance, a fourth of the children would be expected to be correct twice, a half would be (a)

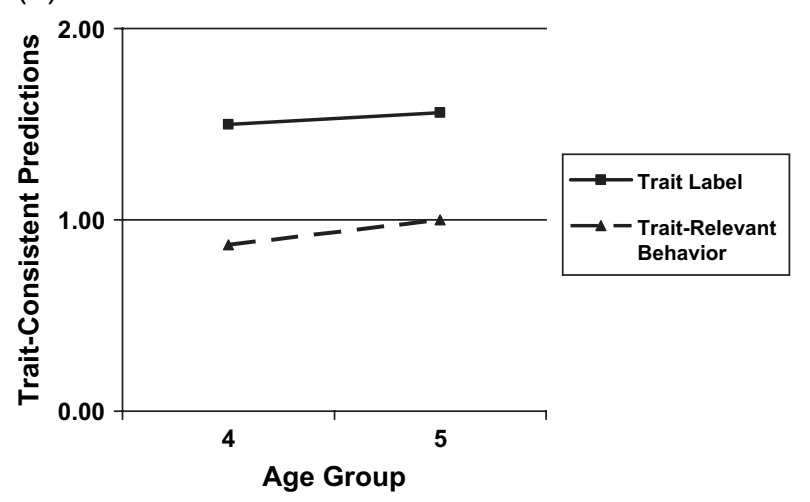

(b)

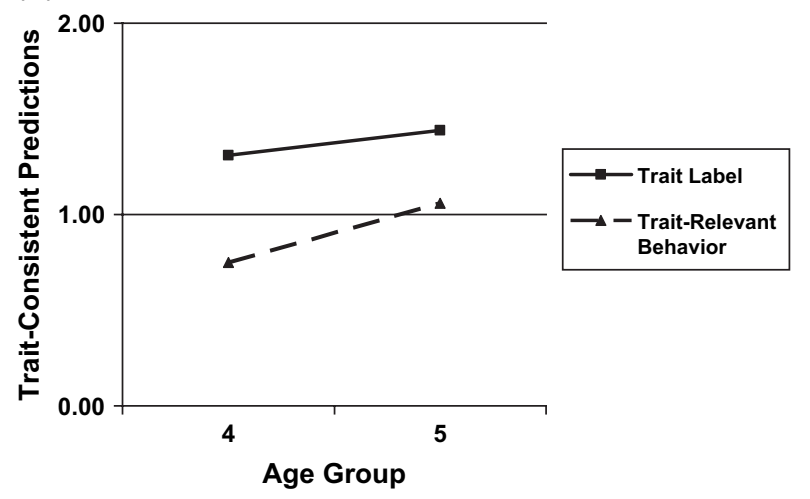

Figure 5. (a) Trait-consistent Time 1 predictions by age and trait presentation conditions (trait labels vs. trait-relevant behaviors) in Experiment 3. (b) Trait-consistent Time 2 predictions by age and trait presentation conditions (trait labels vs. trait-relevant behaviors) in Experiment 3.

expected to be correct for one of the trials, and a fourth would be expected to be incorrect twice. For the trait labels condition, significantly more 4 - and 5-year-olds were consistent for Time 1 predictions than expected by chance: ten 4-year-olds were consistently correct, four were correct once, and two were incorrect twice, $\chi^{2}(2)=12.00, p<.01$; eleven 5 -year-olds were consistently correct, three were correct once, and two were incorrect twice, $\chi^{2}(2)=16.38, p<.001$. However, for the trait-relevant behaviors condition, 4- and 5-year-olds were inconsistent for Time 1 predictions: four 4-year-olds were consistently correct, six were correct once, and six were incorrect twice, $\chi^{2}(2)=$ $1.50, p>.40$; three 5 -year-olds were consistently correct, ten were correct once, and three were incorrect twice, $\chi^{2}(2)=1.00, p>.50$.

\section{Time 2 Predictions}

As shown in Figure 5b, children were also significantly better at Time 2 predictions when given trait 
labels than when given trait-consistent behaviors, $F(1$, $30)=6.66, p<.05$, and there was neither a main effect nor an interaction effect with age group. Targeted analyses showed that 4-year-olds were significantly better at trait-consistent Time 2 predictions from trait labels than from trait-relevant behaviors, $F(1,15)=$ $4.23, p=.057$. For Time 2 predictions, 4-year-olds' predictions from trait labels were not significantly greater than chance; 5-year-olds' predictions from trait labels were significantly greater than chance, $t(15)=2.41, p<.05$, whereas their predictions from trait-relevant behaviors were not significantly different from chance. Nevertheless, both 4- and 5-year-olds' individual patterns of response showed significantly consistent Time 2 predictions from trait labels: nine 4 -year-olds were consistently correct, three were correct once, and four were incorrect twice, $\chi^{2}(2)=$ 9.38, $p<.01$; nine 5-year-olds were consistently correct, five were correct once, and two were incorrect twice, $\chi^{2}(2)=8.38, p<.05$. However, for the traitrelevant behaviors condition, 4 - and 5 -year-olds were inconsistent for Time 1 predictions: two 4 -year-olds were consistently correct, eight were correct once, and six were incorrect twice, $\chi^{2}(2)=2.00, p>.30$; three 5 year-olds were consistently correct, eleven were correct once, and two were incorrect twice, $\chi^{2}(2)=2.38$, $p>.30$. What's more, of the ten 4 -year-olds and eleven 5 -year-olds who were consistently correct for Time 1 predictions from trait labels, significantly more than expected by chance (i.e., nine 4-year-olds and eight 5 year-olds) went on to make consistently correct Time 2 predictions from trait labels, $\chi^{2}(2)=22.60, p<.001$, and $\chi^{2}(2)=13.91, p<.001$, respectively. This pattern of individual children's predictions from trait labels at two time points, one month apart, demonstrates their understanding of stability across time.

\section{Combined Data Across Experiments 2 and 3}

Children in Experiments 2 and 3 made identical judgments about behaviors at Time 1 (questions were phrased identically). Therefore, utilizing data across both studies provides the opportunity to more comprehensively examine young children's pattern of results with a relatively large number of children (i.e., thirty-two 4-year-olds and thirty-two 5-yearolds). Summed across the studies, of the 4-year-olds, 18 were better in the trait labels condition, 4 were better in the trait-relevant behaviors condition, and 10 were equal in both conditions, sign test, $p<.01$. Of the 5 -year-olds, 19 children were better in the trait labels condition, 6 children were better in the trait-relevant behaviors condition, and 7 children were equal in both conditions, sign test, $p<.05$. Thus, consistent with our developmental hypothesis, young children tended to be better at trait-consistent predictions from trait labels than from trait-relevant behaviors.

Furthermore, across the studies, significantly more 4- and 5-year-olds were consistent for predictions in the trait labels condition than expected by chance: eighteen 4-year-olds were consistently correct, nine were correct once, and five were incorrect twice, $\chi^{2}(2)=16.69, p<.001$; twenty-one 5-year-olds were consistently correct, seven were correct once, and four were incorrect twice, $\chi^{2}(2)=28.19, p<.001$. However, 4- and 5-year-olds were inconsistent for predictions in the trait-relevant behaviors condition: seven 4-year-olds were consistently correct, fifteen were correct once, and ten were incorrect twice, $\chi^{2}(2)$ $=0.69, p>.50$; six 5-year-olds were consistently correct, nineteen were correct once, and seven were incorrect twice, $\chi^{2}(2)=1.19, p>.50$.

\section{Discussion}

The results from Experiment 3 replicated and extended the results from Experiment 2. The 4- and 5 -year-olds were consistent in making trait-consistent predictions when given trait labels, whereas they were inconsistent in making trait-consistent predictions when given trait-relevant behaviors. Furthermore, the results show that young children appropriately predict behaviors that are consistent with trait labels for multiple time points. That 4- and 5-year-olds made trait-to-behavior predictions for multiple time points further underwrites the conclusion that they understand traits as being predictive and stable across time. Indeed, consider a lean interpretation of Experiment 2: perhaps young children take the trait labels (e.g., "Bobby is selfish") to be a transient, nontrait description (Bobby is acting selfish right now) and then infer behavior accordingly for that time point. Such a "transient" understanding of provided trait labels is ruled out in Experiment 3, in which young children appropriately predicted the protagonists' trait-consistent behaviors at two time points, one month apart.

\section{General Discussion}

\section{Components of Trait-Consistent Predictions}

For trait reasoning, we propose that the cognitive process of predicting future behaviors from past behaviors consists of two component processes of (1) behavior-to-trait inferences and (2) trait-to-behavior inferences. We hypothesized that young children 
would be able to make each of these sorts of inferences, yet nonetheless fail at the sort of behavior-tobehavior predictions required in prior research.

In Experiment 1, children as young as 4 years of age made appropriate trait-label judgments and ratings based solely on information about the protagonists' behaviors. In addition, children as young as 4 years of age were able to make trait-to-behavior predictions. In Experiments 2 and 3, they were able to use trait labels to predict future behaviors, and they were better at predicting behaviors from trait labels than from previous behaviors. Therefore, the current research found that young children were able to process both components of the two-component process independently. However, they had great difficulty with processing both components together (i.e., make behavior-to-behavior predictions). Specifically, young children were able to make behavior-to-trait inferences and trait-to-behavior predictions, but, just as in previous studies, they were unable to combine both component processes to make behavior-tobehavior predictions. In our studies, and in other research, children appear to have difficulty putting the two component processes together until about 8 years of age. Why is that? We consider a few, though not necessarily mutually exclusive, possibilities.

One possibility is that there is simply an information-processing difficulty, where young children have less cognitive capacity to process both components together. Only by separating the component processes into smaller, manageable parts can young children demonstrate their trait understanding. A second possibility is that although young children are able to make behavior-to-trait inferences and traitto-behavior inferences, their competence in these component processes is still too tenuous to combine both processes to make behavior-to-behavior predictions. Although the 4-year-olds in our studies were significantly able to make behavior-to-trait inferences and trait-to-behavior predictions, it is possible that over this period, children's ability to handle these component processes solidifies until they are able to combine them.

A third possibility is that the data reflect largely young children's emerging understanding of trait labels. Perhaps children's performance in our research reflects an understanding of trait labels (including the ability to make appropriate inferences about and from them) and no further awareness of trait properties. Or, relatedly, perhaps young children have a somewhat more extensive understanding, but trait labels-as presented to children at one point or another in each of our studies-help young children access their concepts of traits. If children are not asked about, or provided with, trait labels, they may fail to engage in trait reasoning and instead consider overt behaviors or global evaluations. Labeling may focus young children's attention on the stable and enduring nature of trait-relevant behaviors. In many areas of cognitive development research, words are considered to provide substantial information about kinds and their richly structured, enduring, and stable nature (Gelman, 2003; Markman, 1989; Waxman, 1999). Labeling is a powerful way of conveying to children category membership and the potential for inferences that go beyond surface appearances (Gelman \& Coley, 1990; Gelman \& Markman, 1986). For examples, Gelman and Heyman (1999) showed that, even for young children, novel trait labels produce judgments of stability. The experimenters described behavioral characteristics to children with either a novel label (e.g., "She is a carrot-eater") or a descriptive phrase (e.g., "She eats carrots whenever she can") and then asked the children to judge the stability of the characteristics. In that study, children as young as 5 years of age judged greater stability for characteristics referred to by a label than for characteristics referred to by a descriptive phrase. These studies demonstrate the power of labels to convey category stability to young children.

At present, our data do not resolve among these different interpretations, separately or in combination. Because research in many areas of cognitive development has shown the power of labels to convey category stability to young children, we favor the interpretation that trait labels help young children to access their conceptions of traits. Nevertheless, further research is needed to examine whether the later development of the ability to make behavior-tobehavior predictions is a result of general informationprocessing difficulty, being not yet fully competent in the component processes, or an early understanding of traits as causal, stable, dispositional entities that is particularly tied to an emerging understanding of trait labels.

\section{Global Evaluations Versus Trait Reasoning}

Our data indicate that a purely global, evaluative interpretation does not account for young children's understanding of traits. Ruble and Dweck (1995) suggest that, when young children make correct judgments on trait tasks, they may simply be inferring behaviors based on their global evaluations of whether they like a person or not, and thus young children do not have a deeper understanding of traits as causal, psychological entities that are stable and internal. We believe that although young children do 
at times use global evaluations to predict behaviors, this does not preclude them from also understanding traits and engaging in trait reasoning. Young children may have a conception of traits but also rely on evaluations to make inferences, especially when the traits involved are highly valenced.

Experiment 1 showed that children did not make global evaluations for all trait-relevant behaviors. In particular, young children did not judge behaviors relating to shyness either positively or negatively. Indeed, in Experiment 1, children's trait-label ratings and evaluative ratings for shyness were not correlated. Furthermore, children's evaluative ratings did not account for all the variance of their trait-tobehavior inferences. These findings suggest that the young children did not merely use global evaluations to make behavior-to-trait inferences in Experiment 1, but instead engaged in trait reasoning.

In Experiments 2 and 3, children showed an understanding of the link from traits to behaviors. Previous research has found trait-consistent predictions especially difficult for young children. However, by making trait-to-behavior predictions, the young children in Experiments 2 and 3 demonstrated at least some aspects of trait-consistent predictions. By using trait labels to predict future behaviors, the children showed an understanding of these labels as referring to dispositional entities important for predicting behaviors across time. Overall, young children demonstrated a nascent understanding of traits as dispositional links to behavior that are stable across time.

\section{Further Issues}

Our manipulation in Experiment 1 of the number of example trait-relevant behaviors did not affect children's trait inferences. It is possible that this manipulation was not large enough to induce an effect or that the number of example trait-relevant behaviors affects not children's judgments but their confidence in their judgments. The many potential effects of greater examples of behavior are open for further research. In addition, the larger issue of designing testing materials to reflect more closely children's everyday social situations is an important one and should be pursued in future research. One line of research to pursue would be children's understanding of the dispositions of people they are familiar with and have known for some time. It might be that young children, if asked to make predictions about people they are familiar with, instead of story characters they only have one or two pieces of information about, would demonstrate still greater trait understanding. With more naturalistic materials, it may be possible to examine whether children younger than 4 years of age have the crucial components of trait understanding.

It is important to note that the type of folk psychology characterized in the current study, unlike theory of mind, is highly variable across cultures. Although the concept of dispositional traits is central to Western individualistic folk psychology, it is not central to adult explanations of behavior in many other cultures (Lillard, 1998; Markus \& Kitayama, 1991; Miller, 1984; Norenzayan, Choi, \& Nisbett, 1999; Shweder \& Bourne, 1984). In future research, it will be important to study cultural variations in developmental trajectories of trait attribution. Miller (1984) demonstrated cultural variations in how older children (8 years and above) use traits to explain behaviors. However, we do not know of any study that has examined cultural variations in the development of the type of trait understanding abilities discussed in this article (e.g., trait-consistent behavior predictions).

Although it appears that behavior-to-behavior predictions are late developing, the current study found that young American children possess aspects of trait understanding that they can use in their everyday social interactions, including some appreciation of dispositional traits and their stability over time. In their everyday life, young children hear trait labels applied to other people. Our data demonstrate that children can infer future behaviors of those people from such information. Furthermore, in their everyday life, young children observe other people's behaviors. Our data suggest that children can, at times, infer and apply trait labels to those people, from such information. Therefore, all in all, by preschool age, children's naïve theories of the social world include traits in their everyday explanations and predictions of people's behaviors and thoughts. This means that in addition to developing a theory of mind, young children, at least in the United States, are well on their way to developing a folk psychology of traits. Future research is needed to investigate the relationship between young children's developing trait understanding and their developing theory of mind. By 18 months of age, infants understand the subjective nature of desires (Repacholi \& Gopnik, 1997). Arguably, many traits are an extension of desires across time and situations (e.g., "Sally wants ice cream" is a desire that, if extended across time and situation, becomes a trait-like preference, "Sally likes ice cream"). Lastly, it is important that further research investigate the extent to which children of different ages understand the causal nature of traits (i.e., that traits cause behaviors). 


\section{Conclusions}

Preschool-age children have a nascent understanding of traits. This understanding appears in a graded, or stepwise fashion, rather than the all-or-none acquisition sometimes described by prior researchers. We propose that predicting future behaviors from past behaviors consists of two component processes: behavior-to-trait inferences and trait-to-behavior inferences. This proposal allows us to address this developmental progression more systematically and resolves inconsistencies in the literature. Counter to previous claims, the current research demonstrates that, in some situations, young children can make trait-consistent behavior predictions. We show that young children can use trait labels, both in inferring traits and in predicting behaviors. However, young children do not appear to make use of trait labels spontaneously to predict future behaviors from past behaviors. Nonetheless, young children's understanding of traits and behaviors in relation to trait labels is a crucial aspect of a conception of traits and is possibly a critical building block in their developing naïve theories of traits and of the social world, in general.

\section{References}

Adolphs, R. (1999). Social cognition and the human brain. Trends in Cognitive Sciences, 3, 469-479.

Alvarez, J. M., Ruble, D. N., \& Bolger, N. (2001). Trait understanding or evaluative reasoning? An analysis of children's behavioral predictions. Child Development, 72, $1409-1425$.

Barenboim, C. (1981). The development of person perception in childhood and adolescence: From behavioral comparisons to psychological constructs to psychological comparisons. Child Development, 52, 129-144.

Berndt, T. J., \& Heller, K. A. (1986). Gender stereotypes and social inferences: A developmental study. Journal of Personality and Social Psychology, 50, 889-898.

Boseovski, J. J., \& Lee, K. (2006). Children's use of frequency information for trait categorization and behavioral prediction. Developmental Psychology, 42, 500-513.

Cain, K. M., \& Dweck, C. S. (1995). The relation between motivational patterns and achievement cognitions through the elementary school years. Merrill-Palmer Quarterly, 41, 25-52.

Cain, K. M., Heyman, G. D., \& Walker, M. E. (1997). Preschoolers' ability to make dispositional predictions within and across domains. Social Development, 6, 53-75.

Camhy, M., \& Ruble, D. N. (1994). Information-seeking during acquaintanceship: Effects of level of social understanding and personal relevance. Social Development, 3, $89-107$.
Carpenter, M., Akhtar, N., \& Tomasello, M. (1998). Fourteen- through 18-month-old infants differentially imitate intentional and accidental actions. Infant Behavior and Development, 21, 315-330.

Dunbar, R. I. M. (1993). Coevolution of neocortical size, group size and language in humans. Behavioral and Brain Sciences, 16, 681-735.

Dweck, C. S., Hong, Y., \& Chiu, C. (1993). Implicit theories: Individual differences in the likelihood and meaning of dispositional inference. Personality and Social Psychology Bulletin, 19, 644-656.

Eder, R. A. (1989). The emergent personologist: The structure and content of 3-, 5-, and 7-year-old's concepts of themselves and other persons. Child Development, 60, $1218-1228$.

Eder, R. A. (1990). Uncovering young children's psychological selves: Individual and developmental differences. Child Development, 61, 849-863.

Erdley, C. A., \& Dweck, C. S. (1993). Children's implicit personality theories as predictors of their social judgments. Child Development, 64, 863-878.

Ferguson, T. J., Van Roozendaal, J., \& Rule, B. G. (1986). Informational basis for children's impressions of others. Developmental Psychology, 22, 335-341.

Flavell, J. H. (1977). Cognitive development. New York: Prentice-Hall.

Flavell, J. H., \& Miller, P. H. (1998). Social cognition. In D. Kuhn \& R. S. Siegler (Eds.), Handbook of child psychology: Vol. 2. Cognition, perception, and language (pp. 851-898). New York: Wiley.

Gelman, S. A. (2003). The essential child: Origins of essentialism in everyday thought. New York: Oxford University Press.

Gelman, S. A., \& Coley, J. D. (1990). The importance of knowing a dodo is a bird: Categories and inferences in 2-year-old children. Developmental Psychology, 26, $796-804$.

Gelman, S. A., Coley, J. D., \& Gottfried, G. M. (1994). Essentialist beliefs in children: The acquisition of concepts and theories. In L. A. Hirschfeld \& S. A. Gelman (Eds.), Mapping the mind: Domain specificity in cognition and culture (pp. 341-365). New York: Cambridge University Press.

Gelman, S. A., \& Heyman, G. D. (1999). Carrot-eaters and creature-believers: The effects of lexicalization on children's inferences about social categories. Psychological Science, 10, 489-493.

Gelman, S. A., \& Markman, E. M. (1986). Categories and induction in young children. Cognition, 23, 183-209.

Gergely, G., Nadasdy, Z., Csibra, G., \& Biro, S. (1995). Taking the intentional stance at 12 months of age. Cognition, 56, 165-193.

Gilbert, D. T. (1998). Ordinary personology. In D. T. Gilbert, S. T. Fiske, G. Lindzey (Eds.), The handbook of social psychology (Vol. 2, pp. 89-150). New York: McGraw-Hill.

Gilbert, D. T., \& Malone, P. S. (1995). The correspondence bias. Psychological Bulletin, 117, 21-38. 
Gnepp, J., \& Chilamkurti, C. (1988). Children's use of personality attributions to predict other people's emotional and behavioral reactions. Child Development, 59, 743-754.

Heider, F. (1958). The psychology of interpersonal relations. New York: Wiley.

Heller, K. A., \& Berndt, T. J. (1981). Developmental changes in the formation and organization of personality attributions. Child Development, 52, 683-691.

Heyman, G. D., \& Dweck, C. S. (1998). Children's thinking about traits: Implications for judgments of self and others. Child Development, 69, 391-403.

Heyman, G. D., \& Gelman, S. A. (1999). The use of trait labels on making psychological inferences. Child Development, 70, 604-619.

Heyman, G. D., \& Gelman, S. A. (2000). Preschool children's use of trait labels to make inductive inferences. Journal of Experimental Child Psychology, 77, 1-19.

Jones, E. E. (1979). The rocky road from acts to dispositions. American Psychologists, 34, 107-117.

Kalish, C. W. (2002). Children's predictions of consistency in people's actions. Cognition, 84, 237-265.

Kelley, H. H. (1973). The process of causal attribution. American Psychologist, 28, 107-128.

Lillard, A. (1998). Ethnopsychologies: Cultural variations in theories of mind. Psychological Bulletin, 123, 1-32.

Livesley, W. J., \& Bromley, D. B. (1973). Person perception in childhood and adolescence. London: Wiley.

Markman, E. M. (1989). Categorization and naming in children. Cambridge, MA: MIT Press.

Markus, H. R., \& Kitayama, S. (1991). Culture and the self: Implications for cognition, emotion, and motivation. Psychological Review, 98, 224-253.

Meltzoff, A. N. (1995). Understanding the intentions of others: Re-enactment of intended acts by 18-month-old children. Developmental Psychology, 31, 838-850.

Miller, J. G. (1984). Culture and the development of everyday social explanation. Journal of Personality and Social Psychology, 46, $961-978$.

Miller, P. H., \& Aloise, R. R. (1989). Young children's understanding of the psychological causes of behavior: A review. Child Development, 60, 257-285.

Nisbett, R. E., \& Ross, L. (1980). Human inference: Strategies and shortcomings of social judgment. Englewood Cliffs, NJ: Prentice Hall.

Norenzayan, A., Choi, I., \& Nisbett, R. E. (1999). Eastern and Western perceptions of causality for social behavior: Lay theories about personalities and situations. In D. A. Prentice \& D. T. Miller (Eds.), Cultural divides: Understanding and overcoming group conflict (pp. 239-272). New York: Russell Sage Foundation.

Peevers, B. H., \& Secord, P. F. (1973). Developmental changes in attribution of descriptive concepts to persons. Journal of Personality and Social Psychology, 27, 120128.

Repacholi, B. M., \& Gopnik, A. (1997). Early reasoning about desires: Evidence from 14- and 18-month-olds. Developmental Psychology, 33, 12-21.
Rholes, W. S., Newman, L. S., \& Ruble, D. N. (1990). Understanding self and other: Developmental and motivational aspects of perceiving others in terms of invariant dispositions. In E. T. Higgins \& R. Sorrentino (Eds.), Handbook of motivation and cognition: Vol. 2. Foundations of social behavior (pp. 369-407). New York: Guilford Press.

Rholes, W. S., \& Ruble, D. N. (1984). Children's understanding of dispositional characteristics of others. Child Development, 55, 550-560.

Ross, L. (1977). The intuitive psychologist and his shortcomings. In L. Berkowitz (Ed.), Advances in experimental social psychology: Vol. 10 (pp. 173-220). New York: Academic Press.

Rotenberg, K. J. (1980). Children's use of intentionality in judgments of character and disposition. Child Development, 51, 282-284.

Rotenberg, K. J. (1982). Development of character constancy of self and other. Child Development, 53, 505-515.

Ruble, D. N., \& Dweck, C. S. (1995). Self-conceptions, person conceptions, and their development. In N. Eisenberg (Ed.), Review of personality and social psychology: Vol. 15. Social development (pp. 109-139). Thousand Oaks, CA: Sage.

Shantz, C. U. (1983). Social cognition. In J. H. Flavell \& E. M. Markman (Eds.), The handbook of child psychology, vol. III, cognitive development (pp. 495-555). New York: Wiley.

Shweder, R. A., \& Bourne, E. J. (1984). Does the concept of the person vary cross-culturally? In R. A. Shweder \& R. A. LeVine (Eds.), Culture theory: Essays on mind, self, and emotion. Cambridge, UK: Cambridge University Press.

Stipek, D. J., \& Daniels, D. H. (1990). Children's use of dispositional attributions in predicting the performance and behavior of classmates. Journal of Applied Developmental Psychology, 11, 13-28.

Taylor, M. (1996). A theory of mind perspective on social cognitive development. In R. Gelman \& T. K. Au (Eds.), Handbook of perception and cognition: Perceptual and cognitive development. New York: Academic Press.

Tomasello, M. (1999). The cultural origins of human cognition. Cambridge, MA: Harvard University Press.

Trope, Y., \& Higgins, E. T. (1993). The what, when, and how of dispositional inference: New answers and new questions. Personality and Social Psychology Bulletin, 19, 493-500.

Waxman, S. R. (1999). The dubbing ceremony revisited: Object naming and categorization in infancy and early childhood. In D. L. Medin \& S. Atran (Eds.), Folkbiology (pp. 233-284). Cambridge, MA: MIT Press.

Weiner, B. (Ed.). (1974). Achievement motivation and attribution theory. Morristown, NJ: General Learning Press.

Wellman, H. M. (1990). The child's theory of mind. Cambridge, MA: MIT Press.

Wellman, H. M., Cross, D., \& Watson, J. (2001). Metaanalysis of theory of mind development: The truth about false belief. Child Development, 72, 655-684.

Wellman, H. M., \& Gelman, S. A. (1998). Knowledge acquisition in foundational domains. In D. Kuhn \& R. S. Siegler (Eds.), Handbook of child psychology: Vol. 2. 
Cognition, perception, and language (pp. 523-573). New York: Wiley.

Wellman, H. M., \& Woolley, J. D. (1990). From simple desires to ordinary beliefs: The early development of everyday psychology. Cognition, 35, 245-275.

Wellman, H. M., \& Liu, D. (2004). Scaling of theory-ofmind tasks. Child Development, 75, 523-541.

Whiten, A., \& Byrne, R. W. (1997). Machiavellian intelligence II: Evaluations and extensions. Cambridge, UK: Cambridge University Press.

Yuill, N. (1992). Children's conception of personality traits. Human Development, 35, 265-279.

Yuill, N., \& Pearson, A. (1998). The development of bases for trait attribution: Children's understanding of traits as causal mechanisms based on desire. Developmental Psychology, 34, 574-586.

\section{Appendix A: Experiment 1: Trait-Consistent and Trait-Inconsistent Stories}

All three trait-relevant behaviors are provided for the trait-consistent condition of each trait. The traitrelevant behaviors for the trait-inconsistent condition are the opposites of the behaviors in the traitconsistent condition, and thus only one behavior is provided, as an example, for the trait-inconsistent condition of each trait.

\section{Selfish Trait-Consistent}

(1) Here's Bobby in school. He has a bag of cookies. One of his classmates does not have a lunch to eat and she asks Bobby for some cookies. Look, Bobby does not give her any of his cookies. (2) Here's Bobby at the playground. He is blowing big bubbles. One of the other children does not have bubbles and she asks Bobby to let her blow bubbles too. Look, Bobby does not let her blow bubbles. (3) Here's Bobby in class. He has one of his toys for show-and-tell. One of the other children has never played with that toy and she asks Bobby to let her play with it. Look, Bobby does not let her play with the toy.

\section{Selfish Trait-Inconsistent}

(1) Here's Bobby in school. He has a bag of cookies. One of his classmates does not have a lunch to eat and she asks Bobby for some cookies. Look, Bobby gives her some of his cookies.

\section{Nice Trait-Consistent}

(1) Here's Johnny on the street. He is going to the ice cream shop. An old woman is having a lot of trouble carrying a big heavy box. Look, Johnny carries the woman's box and takes it to her door. (2) Here's Johnny at the dining room table. He is playing with his toys. His mom says it's time for dinner. Look, Johnny cleans up right away and sets the table for dinner. (3) Here's Johnny at the store. He is buying some candy. A woman accidentally knocks over a cereal box. Look, Johnny picks up the cereal box and stacks it back up.

\section{Nice Trait-Inconsistent}

(1) Here's Johnny on the street. He is going to the ice cream shop. An old woman is having a lot of trouble carrying a big heavy box. Look, Johnny does not carry the woman's box and just goes to the ice cream shop.

\section{Mean Trait-Consistent}

(1) Here's Mary in the living room. She is watching TV. The family's cat is in the room. Look, Mary pulls hard on the cat's tail and the cat runs away. (2) Here's Mary at home. She is going up the stairs. Her little brother has left his blocks in the stairway. Look, Mary kicks his blocks down the stairs and walks to her room. (3) Here's Mary at school. She is walking down the hall. She walks by a boy carrying a plant. Look, Mary pushes the boy and his plant falls on the floor and breaks.

\section{Mean Trait-Inconsistent}

(1) Here's Mary in the living room. She is watching TV. The family's cat is in the room. Look, Mary looks at the cat and then keeps watching her show.

\section{Shy Trait-Consistent}

(1) Here's Tracy at the library. She is watching a puppet show. The puppet man asks for a child helper to come up on stage. Look, Tracy just looks down and does not raise her hand. (2) Here's Tracy at her grandma's house. She is having dinner with her relatives. Her aunts, uncles, and cousins are all at the dinner table talking to each other. Look, Tracy just sits there quietly and does not talk to anyone. (3) Here's Tracy at her new school. It is her first day at school. The teacher asks Tracy to tell everyone something interesting about herself. Look, Tracy only tells them her name and sits down right away.

\section{Shy Trait-Inconsistent}

(1) Here's Tracy at the library. She is watching a puppet show. The puppet man asks for a child 
helper to come up on stage. Look, Tracy jumps up and raises her hand.

\section{Appendix B: Experiment 2 (and Experiment 3 Time 1) Trait-Relevant Situation and Prediction Choices}

\section{Selfish Situation and Prediction Choices}

One day Bobby sees his younger sister playing with his toys in his room. What does Bobby do when he sees his sister playing with his toys? Does he stop her from playing with his toys? Or does he let her keep playing and give her more of his toys to play with?

\section{Nice Situation and Prediction Choices}

One day Johnny goes to his aunt's house to play with his cousins, and when he gets there, they are all cleaning up their toys. What does Johnny do when he sees them cleaning up their toys? Does he stay and clean up the toys? Or does he not help clean up and goes home?

\section{Mean Situation and Prediction Choices}

One day Mary is walking to the playground and sees a boy trip, fall, and cry. What does Mary do when she sees the boy fall and cry? Does she laugh at and make fun of the boy? Or does she help the boy get up?

\section{Shy Situation and Prediction Choices}

One day Tracy goes to camp for the first time and there are a lot of new children she has never met before. What does Tracy do when she sees all the children? Does she stay quiet and not talk to anyone? Or does she go around meeting new children, talking to everyone? 